\title{
Présence, populations et dégâts de l'alucite des céréales Sitotroga cerealella (Olivier) (Lepidoptera, Gelechiidae) sur les stocks de riz au Bénin*
}

\author{
Abou Togola ${ }^{1}$ \\ Francis E. Nwilene ${ }^{2}$ \\ Daniel C. Chougourou ${ }^{3}$ \\ Tolulope Agunbiade ${ }^{2}$ \\ ${ }^{1}$ Centre du riz pour l'Afrique (AfricaRice) \\ Division Entomologie \\ 01 BP 2031 \\ Cotonou \\ Bénin \\ $<$ a.togola@cgiar.org> \\ ${ }^{2}$ Centre du riz pour l'Afrique (AfricaRice) \\ PMB 5320 Oyo Road \\ Ibadan \\ Nigeria \\ $<$ f.nwilene@cgiar.org> \\ $<$ tolulope_adeniran@yahoo.com> \\ 3 École polytechnique d'Abomey Calavi (Epac) \\ 01 BP 2009 \\ Cotonou \\ Bénin \\ <chougouroud@yahoo.de>
}

\begin{abstract}
Résumé
L'alucite des céréales, Sitotroga cerealella (Olivier) (Lepidoptera, Gelechiidae), est un ravageur présent sur plusieurs continents, qui cause des dégâts importants sur les stocks de diverses céréales (riz, maïs, sorgho, etc.). Cette étude visait à analyser la présence de S. cerealella dans des zones de forte production de riz au Bénin, et à évaluer le niveau des populations du ravageur et les dégâts qu'il occasionne sur les stocks de riz paysans. À cet effet, des échantillons de riz paddy ont été collectés sur 11 sites de stockage paysan. Des pièges à phéromones ont aussi été placés dans des champs de riz pour étudier l'origine des infestations. Les résultats ont montré que $70 \%$ des stocks de riz étaient infestés par $S$. cerealella, avec des populations moyennes variant de 30 à 300 individus par kilogramme de paddy. Dans les échantillons infestés, le niveau des dégâts variait de 3 à $18 \%$ de grains attaqués pour des périodes de stockage allant de deux à quatre mois. Le piégeage a montré que $80 \%$ des champs étaient infestés, avec une capture moyenne de 16 individus par piège. La présence de ce ravageur est donc confirmée au Bénin, et cette étude montre que des dégâts importants peuvent être causés aux stocks de riz dès que la période de stockage s'allonge à trois-quatre mois.
\end{abstract}

Mots clés : Bénin ; dégât ; piège ; phéromone ; riz ; Sitotroga cerealella.

Thèmes : productions végétales; systèmes agraires.

\section{Abstract \\ Presence, populations and damage of the angoumois grain moth, Sitotroga cerealella (Olivier) (Lepidoptera, Gelechiidae), on rice stocks in Benin}

The Angoumois grain moth, Sitotroga cerealella (Olivier) (Lepidoptera, Gelechiidae), is a major pest on several continents causing major damage to stocks of several cereals (rice, maize, sorghum etc.). This study aimed at analyzing the presence of S. cerealella in key sites of rice production in Benin and evaluating the level of the pest population and its damage to farmers' rice stocks. For this purpose, paddy rice samples were collected from 11 sites of peasant stockage. In addition, pheromone traps were placed in farmers' paddy fields to study infestations. The results showed that $70 \%$ of the rice stocks were infested by S. cerealella, with average populations varying from 30 to 300 individuals per $\mathrm{kg}$ of paddy. In infested samples, the damage level ranged from $3 \%$ to $18 \%$ of grains attacked for 2 to 4 months of storage. Pheromone trapping showed that $80 \%$ of the rice fields were infested, with an average of 16 individuals captured per trap. The presence of this pest is thus confirmed on rice in Benin. This study also shows that important damage can be caused on stored rice as soon as the storage period reaches 3 to 4 months.

Key words: Benin; damage; pheromone; rice; Sitotroga cerealella; traps.

Subjects: agrarian systems; vegetal productions. 
I alucite des céréales, Sitotroga cerealella (Olivier) (Lepidoptera, Gelechiidae), est un des plus importants ravageurs primaires du riz, du sorgho et du maïs. Il s'attaque également au blé et à l'orge (Teetes et al., 1995). Les chenilles dévorent l'albumen des grains des céréales qu'elles atteignent en creusant une galerie à travers les glumes (Raoelijaona, 1996). Dans des conditions de forte infestation, les produits stockés peuvent subir $100 \%$ de pertes (Mele, EPAC, 2007, résultats non publiés). Les attaques de $S$. cerealella se traduisent par la diminution du poids des produits, la baisse du pouvoir germinatif des graines et par la perte de la valeur nutritive et de la valeur marchande.

$S$. cerealella est décrit comme un insecte cosmopolite (CAB International, 2005), ravageur primaire de nombreuses céréales. Toutefois, aucune publication ne précise la présence, le niveau des populations et les dégâts de cet insecte sur le riz au Bénin. Sa présence a été signalée sur des stocks paysans de maïs, notamment dans les savanes guinéennes du pays (Gbati et al., 2002). Cette même étude a indiqué la disparition de l'insecte suite à l'introduction d'ennemis naturels. Nykjaer et al., 2002 ont signalé l'absence de $S$. cerealella au sein de l'entomofaune collectée sur les stocks de maïs dans cette région.

Jusqu'à récemment, l'alucite des céréales, à l'instar d'autres insectes des stocks, ne représentait pas un problème majeur pour le riz au Bénin, car la production rizicole était insuffisante pour être conservée pendant plus de trois mois (Mele, EPAC, 2007 ; résultats non publiés). Dans ces conditions, les risques d'infestation des stocks étaient minimes, d'autant que la riziculture n'était pratiquée que par quelques communautés rurales. Actuellement, le riz prend une place importante dans les habitudes alimentaires béninoises, ce qui fait que sa culture s'intensifie et s'étend (MAEP/DFRN, 2000). De plus, les échanges de produits céréaliers, dans le pays et à travers les frontières, constituent des voies de dissémination possibles des ravageurs. Aujourd'hui, l'infestation des stocks de riz par l'alucite apparaît comme un problème préoccupant dans les zones rizicoles du pays, sans que des études scientifiques aient été menées pour déterminer la présence du ravageur, le niveau des populations, ou les dégâts causés.

La présente étude vise à analyser la présence de $S$. cerealella dans les zones rizicoles du Bénin, à établir le niveau des populations dans le riz stocké en milieu paysan et à évaluer les dégâts au cours du stockage.

\section{Matériel et méthode}

Au cours des campagnes 2007 et 2008, des investigations ont été menées par l'unité d'entomologie du Centre du riz pour l'Afrique (AfricaRice) dans 11 localités rizicoles du Bénin: Niaouli (commune d'Alladah), Bohicon (commune de Bohicon), Koussin et Lélé (commune de Covè), Eglimé (commune d'Aplahoué), Dévé (commune de Dogbo), Dokparou (commune de Parakou), Sowé (commune de Glazoué), Houeda (commune d'Adjohoun), Malanville (commune de Malanville) et Togoudo (station de l'Institut international pour l'agriculture tropicale [IITA]). Ce sont des zones de forte production de riz, où les producteurs disposent de stocks de paddy destinés à la consommation ou aux semences. Au cours des campagnes 2007 et 2008, 33 réserves de paddy ont été identifiées, soit 3 par site. L'échantillonnage a porté sur des stocks de deux, trois, et quatre mois, car les réserves de durée supérieure à un mois sont plus favorables aux infestations de l'alucite (Mele, EPAC, 2007, résultats non publiés). Deux modes de stockages étaient utilisés par les paysans chez qui ont été réalisés les prélèvements : dans un magasin fermé (le plus fréquent) et sous hangar ouvert. Les stocks de riz paddy non traités ont été ciblés. Le mode de stockage et les variétés de riz ont été enregistrés afin d'étudier leur influence éventuelle. Un échantillon d'un kilogramme de riz a été prélevé à chaque fois. Les échantillons ont été conditionnés dans des sachets plastiques et placés dans un congélateur pendant 24 heures afin de tuer les insectes présents. Ils ont été ensuite analysés afin de noter les populations de $S$. cerealella et d'évaluer les dégâts. La technique de flottaison a permis de séparer les grains attaqués des grains sains et de repérer les formes larvaires cachées. Les grains attaqués par $S$. cerealella ont été identifiés en les examinant à la loupe pour voir le contour des trous ou localiser les chenilles à l'intérieur. Afin de situer l'origine des infestations, des pièges à phéromones adaptés à cette espèce ont été placés en trois répétitions dans des parcelles de riz (en maturation) choisies au hasard.
Les pièges étaient installés le soir et dépouillés le lendemain dans la matinée. Dans les zones étudiées, la majeure partie des paysans cultivait des variétés locales de l'espèce Oryza glaberrima (riz africain), tandis qu'une minorité cultivait des variétés améliorées Toss-long et INARIS (Oryza sativa). Une autre variété d'O. sativa (riz asiatique) présente était celle du site de la station de recherche d'AfricaRice à Togoudo.

Les données collectées ont concerné les populations de $S$. cerealella (cumul du nombre de larves et d'adultes) sur les échantillons de riz stocké, les populations d'adultes attrapées aux pièges à phéromones et le nombre de grains attaqués. Ce dernier paramètre a permis de calculer le pourcentage de paddy attaqué. Les données ont été soumises à l'analyse de variance, modèle mixte à trois facteurs (durée de stockage, mode de stockage et variété de riz), au moyen du logiciel SAS/PC version 9.1 (SAS Institute, 2002-2003). Les moyennes obtenues ont été comparées par le test de StudentNewman-Keuls. Des coefficients de corrélation ont été calculés pour déterminer le degré de lien d'une part entre le nombre de $S$. cerealella et le pourcentage de grains attaqués, d'autre part entre ces deux paramètres et la durée et le mode de stockage.

L'identification des spécimens (larves et adultes) a été effectuée au laboratoire du Centre des ressources de biodiversité de l'IITA au Bénin.

\section{Résultats et discussion}

\section{Occurrence des populations de $S$. cerealella sur les stocks de riz}

Dans la plupart des sites explorés, S. cerealella est présent dans le riz. De 2007 à 2008, on a noté une augmentation moyenne de $22 \mathrm{~S}$. cerealella par kilogramme de paddy, soit $18 \%$ (tableau 1). Pour les populations d'adultes capturés dans les champs, on a noté une augmentation moyenne de trois individus par piège, soit 19\% (tableau 1). Environ $70 \%$ des stocks de riz échantillonnés au cours de ces deux années se sont avéré être infestés par ce ravageur, avec des densités moyennes allant selon les sites de 30 à 300 insectes (larves et adultes) par kilogramme de paddy (tableau 2). 
Tableau 1. Populations et dégâts de Sitotroga cerealella sur le riz à travers les sites observés en 2007 et 2008 au Bénin.

Table 1. Populations and damage of Sitotroga cerealella on rice on sites studied in 2007 and 2008 in Benin.

\begin{tabular}{|c|c|c|c|c|c|c|c|}
\hline Année & Sites d'étude & $\begin{array}{l}\text { Durée } \\
\text { de stockage } \\
\text { (mois) }\end{array}$ & $\begin{array}{l}\text { Mode } \\
\text { de stockage }\end{array}$ & $\begin{array}{l}\text { Variétés } \\
\text { de riz stocké }\end{array}$ & $\begin{array}{c}\text { Nombre } \\
\text { de Sitotroga/kg } \\
\text { paddy } \\
\text { (larves } \\
\text { et adultes) }\end{array}$ & $\begin{array}{c}\text { Nombre } \\
\text { de Sitotroga/ } \\
\text { piège } \\
\text { (adultes) }\end{array}$ & $\begin{array}{c}\text { Pourcentages } \\
\text { grains } \\
\text { attaqués }\end{array}$ \\
\hline \multirow[t]{11}{*}{2007} & Houêda & 2 & $M$ & VL & $26 \mathrm{~g}$ & $4 \mathrm{e}$ & 2,5 de \\
\hline & Niaouli & 4 & $\mathrm{M}$ & VL & $212 b$ & $18 \mathrm{bc}$ & 14,3 a \\
\hline & Dévé & 3 & $\mathrm{M}$ & $V L$ & $82 \mathrm{e}$ & $5 e$ & $7,3 \mathrm{c}$ \\
\hline & Bohicon & 2 & $\mathrm{M}$ & INARIS & $38 \mathrm{f}$ & $10 d$ & $3,4 d$ \\
\hline & Sowé & 3 & $\mathrm{M}$ & VL & $\mathrm{Oh}$ & $0 \mathrm{e}$ & $0 \mathrm{e}$ \\
\hline & Malanville & 3 & $M$ & VL & $200 c$ & $21 b$ & $9,3 \mathrm{bc}$ \\
\hline & Eglimé & 4 & $\mathrm{H}$ & VL & $196 c$ & $13 \mathrm{~cd}$ & $10,4 \mathrm{~b}$ \\
\hline & Dokparou & 2 & $\mathrm{H}$ & $\mathrm{VL}$ & $\mathrm{Oh}$ & $0 \mathrm{e}$ & $0 \mathrm{e}$ \\
\hline & Koussin & 2 & $\mathrm{H}$ & Toss-Long & $\mathrm{Oh}$ & $14 \mathrm{~cd}$ & $0 \mathrm{e}$ \\
\hline & Togoudo & 4 & $\mathrm{H}$ & Toss- Long & $256 a$ & $23 \mathrm{~b}$ & 13,4 a \\
\hline & Lélé & 2 & $\mathrm{H}$ & WAB56-50 & $116 d$ & $54 a$ & $6,7 \mathrm{c}$ \\
\hline \multicolumn{2}{|c|}{ Moyennes générales } & & & & 102 & 15 & 6,1 \\
\hline \multirow[t]{11}{*}{2008} & Houêda & 2 & $M$ & VL & $31 \mathrm{f}$ & $12 d$ & 2,9 ef \\
\hline & Niaouli & 4 & $M$ & VL & $224 b$ & $51 \mathrm{~b}$ & $12,7 \mathrm{c}$ \\
\hline & Dévé & 3 & $\mathrm{M}$ & VL & $88 d$ & $4 \mathrm{fg}$ & $9,8 \mathrm{~cd}$ \\
\hline & Bohicon & 2 & M & INARIS & $54 \mathrm{e}$ & $35 \mathrm{~b}$ & $6 \mathrm{de}$ \\
\hline & Sowé & 3 & $M$ & VL & $0 \mathrm{~g}$ & $0 \mathrm{~g}$ & $0 \mathrm{f}$ \\
\hline & Malanville & 3 & $\mathrm{M}$ & VL & $188 \mathrm{c}$ & $10 \mathrm{de}$ & $13,3 \mathrm{c}$ \\
\hline & Eglimé & 4 & $\mathrm{H}$ & VL & 338 a & $22 \mathrm{c}$ & $18 \mathrm{~b}$ \\
\hline & Dokparou & 2 & $\mathrm{H}$ & VL & $0 \mathrm{~g}$ & $0 \mathrm{~g}$ & $0 f$ \\
\hline & Koussin & 2 & $\mathrm{H}$ & Toss-Long & $0 \mathrm{~g}$ & $21 \mathrm{c}$ & $0 f$ \\
\hline & Togoudo & 4 & $\mathrm{H}$ & Toss- Long & $354 \mathrm{a}$ & $37 b$ & 23,3 a \\
\hline & Lélé & 2 & $\mathrm{H}$ & WAB56-50 & $92 d$ & 7 ef & $9,8 \mathrm{~cd}$ \\
\hline \multicolumn{2}{|c|}{ Moyennes } & & & & 124 & 18 & 8,7 \\
\hline
\end{tabular}

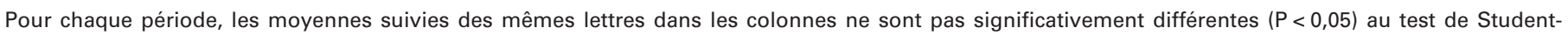
Newman-Keuls.

$\mathrm{M}$ : magasin, $\mathrm{H}$ : hangar, $\mathrm{VL}$ : variété locale (variété indigène du paysan).

La présence et le niveau des populations de $S$. cerealella sur le riz stocké ont varié suivant la durée et le mode de stockage, les sites et les variétés de riz concernées. Les sites de Togoudo, Eglimé, Niaouli et de Malanville ont enregistré les plus fortes populations (respectivement 305, 267, 218 et 194 S. cerealella par kilogramme de paddy). Il n'y a que dans les sites de Koussin, Sowé et Dokparou qu'aucune forme du ravageur n'a été trouvée sur les stocks (tableau 2). Les caractéristiques agroclimatiques des différentes régions pourraient expliquer ces différences. En effet, dans les localités côtières du Sud, l'humidité relative est plus élevée (80-95\%) et la température oscille entre 20 et $30^{\circ} \mathrm{C}$. Or, le développement des populations de $S$. cerealella est maximum dans cette gamme de températures et d'humidité (Hansen et al., 2002) et les femelles sont plus fertiles (Hansen et al., 2004). Les climats humides entraînent aussi une augmentation de la teneur en eau des paddy stockés, créant un environnement favorable à la multiplication de l'alucite (Ratnadass et Sauphanor, 1989). Cela rejoint les observations d'Arbogast et al. (1997) sur l'influence de l'état du produit stocké et des conditions climatiques sur les attaques de $S$. cerealella. Enfin, les régions sud sont des zones de forts échanges céréaliers avec les pays voisins, ce qui peut accroitre la population du ravageur. Pour la zone de Malanville, localité du Nord, cela peut être lié au fait que c'est une zone de grande production de riz irrigué en toute saison, ce qui peut permettre à l'insecte de se multiplier d'autant plus aisément qu'il peut venir des champs (Teetes et al., 1995). En outre, Malanville est une ville frontalière qui reçoit des céréales venant d'autres pays susceptibles d'abriter le ravageur.

Une présence plus abondante de S. cerealella a été notée sur les stocks de 4 mois (263 alucites par kg de riz). Sur les stocks de 2 et 3 mois, les populations moyennes enregistrées ont été respectivement de 36 et 96 alucites par kg de riz. Les stocks conservés sous hangar ouvert se sont montrés un peu plus infestés que ceux gardés dans des magasins fermés, avec des populations moyennes respectives de 135 et 95 alucites par $\mathrm{kg}$ de grain (différences non significatives). En ce qui concerne les variétés de riz, les populations les plus nombreuses ont été enregistrées sur WAB 56-50 et les variétés 
Tableau 2. Moyennes des populations et dégâts $(2007,2008)$ de Sitotroga cerealella sur le riz à travers les sites observés.

Table 2. Mean populations and damage $(2007,2008)$ of Sitotroga cerealella on rice in the sites studied.

\begin{tabular}{|c|c|c|c|c|c|c|}
\hline Sites d'étude & $\begin{array}{l}\text { Durée } \\
\text { de stockage } \\
\text { (mois) }\end{array}$ & $\begin{array}{l}\text { Mode } \\
\text { de stockage }\end{array}$ & $\begin{array}{l}\text { Variétés } \\
\text { de riz stocké }\end{array}$ & $\begin{array}{c}\text { Nombre de } \\
\text { Sitotroga/kg paddy } \\
\text { (larves et adultes) }\end{array}$ & $\begin{array}{c}\text { Nombre } \\
\text { de Sitotroga/ } \\
\text { piège (adultes) }\end{array}$ & $\begin{array}{l}\text { Pourcentages } \\
\text { grains attaqués }\end{array}$ \\
\hline Houêda & 2 & $M$ & VL & $29 c$ & $8 a b$ & $2,7 d$ \\
\hline Niaouli & 4 & $M$ & VL & $218 a b$ & $35 a b$ & $13,5 a b$ \\
\hline Dévé & 3 & $M$ & VL & $85 \mathrm{c}$ & $5 a b$ & 8,5 bc \\
\hline Bohicon & 2 & $M$ & INARIS & $46 \mathrm{c}$ & $23 a b$ & $4,7 \mathrm{~cd}$ \\
\hline Sowé & 3 & $\mathrm{M}$ & VL & $0 \mathrm{c}$ & $0 \mathrm{~b}$ & $0 d$ \\
\hline Malanville & 3 & $M$ & VL & $194 \mathrm{~b}$ & $16 a b$ & $11,3 \mathrm{abc}$ \\
\hline Eglimé & 4 & $\mathrm{H}$ & VL & $267 a b$ & $18 a b$ & $14,2 a b$ \\
\hline Dokparou & 2 & $\mathrm{H}$ & VL & O c & $0 \mathrm{~b}$ & $0 \mathrm{~d}$ \\
\hline Koussin & 2 & $\mathrm{H}$ & Toss-Long & $0 \mathrm{c}$ & $18 a b$ & $0 \mathrm{~d}$ \\
\hline Togoudo & 4 & $\mathrm{H}$ & Toss- Long & 305 a & $46 a$ & 18,3 a \\
\hline Lélé & 2 & $\mathrm{H}$ & WAB56-50 & $104 \mathrm{c}$ & $15 a b$ & 8,2 bcd \\
\hline
\end{tabular}

Les moyennes suivies des mêmes lettres dans chaque colonne ne sont pas significativement différentes (P < 0,05$)$ au test de Student-Newman-Keuls.

$\mathrm{M}$ : magasin ; $\mathrm{H}$ : hangar ; $\mathrm{VL}$ : variété locale (variété indigène du paysan).

locales avec respectivement 305 et $113 \mathrm{~S}$. cerealella en moyenne, tandis que les plus faibles populations ont été notées sur les variétés INARIS et Toss-Long avec 46 et 52 individus respectivement (tableau 2), mais ces différences sont difficiles à interpréter compte tenu du lien entre sites et variétés. La présence de $S$. cerealella a été détectée sur $80 \%$ des sites avec les pièges à phéromones. Les niveaux moyens de capture au champ allaient de 0 à 46 individus par site, avec une moyenne générale de 16 adultes. Les localités de Togoudo, Niaouli et Bohicon ont les plus grandes populations moyennes avec respectivement 46, 35 et 23 adultes, tandis qu'aucune présence n'a été enregistrée à Sowé et Dokparou. À Koussin, bien que les stocks de riz n'aient pas été attaqués, le piégeage a révélé que le site était une zone endémique (tableaux 1 et 2).

Une corrélation positive et hautement significative a été notée entre les infestations dans les champs (populations issues de la capture aux pièges à phéromones) et celles du riz stocké $(\mathrm{r}=0,94 ; \mathrm{P}<0,0001)$ (tableau 3). Cela renforce l'hypothèse que la contamination des stocks de riz peut provenir des champs (Teetes et al., 1995), tout comme celle des champs peut provenir des magasins de stockage (Barney et al., 1996). En ce qui concerne les traitements lors du stockage, d'une part, les paysans béninois appliquent très rarement des insecticides et, d'autre part, l'alucite peut développer une résistance à ces produits (Boshra et al., 2007).

\section{Statut des dégâts de S. cerealella sur les stocks de riz}

Les dégâts de $S$. cerealella sont aisément identifiés par la présence de soies, d'excréments et de larves, et par la dimension des trous dans les grains. D'après les résultats obtenus, les dégâts notés sur les paddy ont été de $6,1 \%$ en moyenne en 2007, contre $8,7 \%$ en moyenne en 2008 (+ $42 \%$; tableau 1).

Dans les échantillons infestés, les niveaux des attaques se sont situés en moyenne à

\section{Tableau 3. Corrélations entre les paramètres d'infestation de Sitotroga cerealella.}

Table 3. Correlations between Sitotroga cerealella infestation parameters.

\begin{tabular}{|c|c|c|c|c|}
\hline & \multicolumn{2}{|c|}{$\begin{array}{c}\text { Nombre de Sitotroga/ } \\
\text { kg paddy }\end{array}$} & \multicolumn{2}{|c|}{$\begin{array}{c}\text { Pourcentage } \\
\text { de grains attaqués }\end{array}$} \\
\hline & $\mathbf{r}$ & $\mathbf{s}$ & $\mathbf{r}$ & $\mathbf{s}$ \\
\hline Durée de stockage & 0,82 & THS & 0,76 & THS \\
\hline Mode de stockage & 0,18 & NS & 0,10 & NS \\
\hline Nombre de Sitotroga/kg paddy & & & 0,96 & THS \\
\hline Pourcentage de grains attaqués & 0,96 & THS & & \\
\hline Population par piège & 0,94 & THS & 0,58 & THS \\
\hline
\end{tabular}

$r$ : coefficient de corrélation de Pearson; s : significativité ; NS : non significatif ; THS : très hautement significatif (seuil de 0,0001).
$3 \%$ et $18 \%$, pour respectivement deux et quatre mois de stockage. La figure 1 illustre l'évolution des populations et des dégâts du ravageur en fonction de la durée du stockage.

A été notée une corrélation positive hautement significative entre, d'une part, les pourcentages de grains attaqués et les populations de $S$. cerealella $(r=0,96)$, et, d'autre part, entre les pourcentages de grains attaqués et la durée de stockage $(\mathrm{r}=0,76)$ (tableau 3). Même si les stocks placés dans les magasins ont été un peu moins endommagés (6,7\%) que ceux placés sous les hangars $(8,1 \%)$, l'influence du mode de stockage sur les niveaux de dégâts causés par $S$. cerealella est statistiquement non significative. 


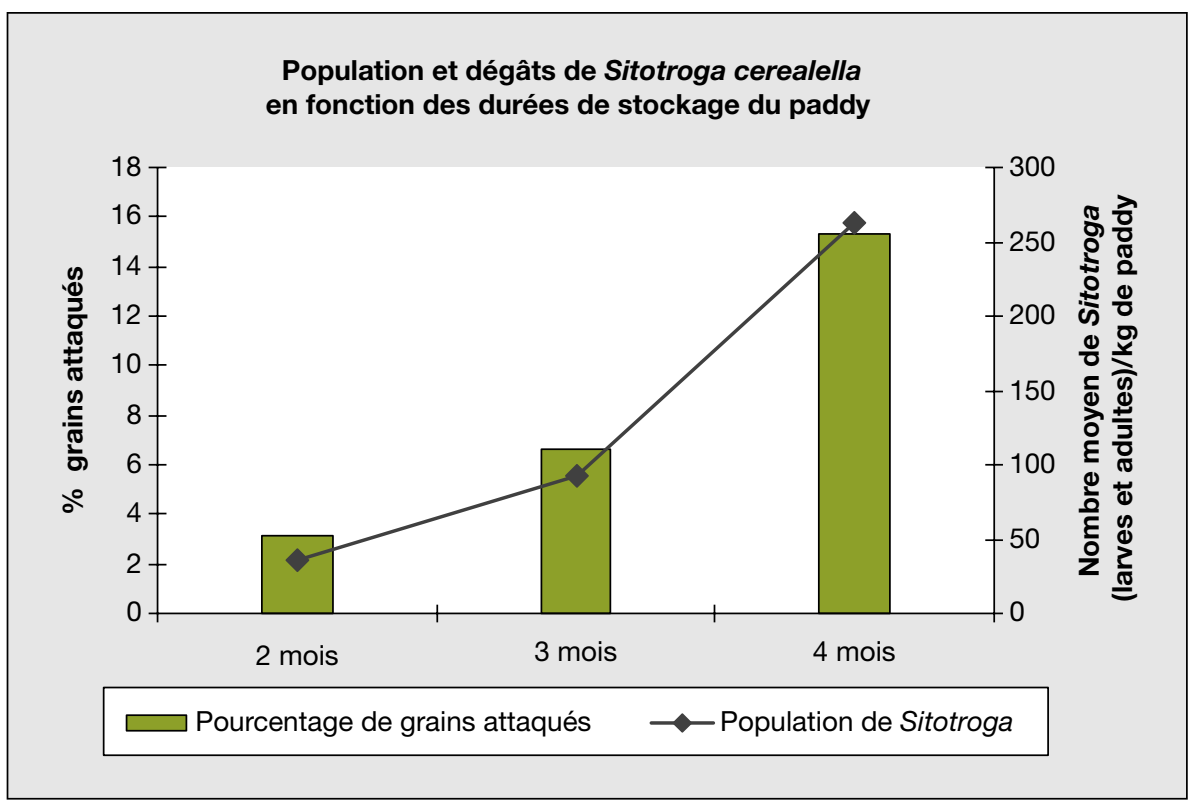

Figure 1. Populations et dégâts de Sitotroga cerealella en fonction des durées de stockage.

Figure 1. Populations and damage of Sitotroga cerealella with reference to the duration of storage.

Les niveaux des dégâts enregistrés sont très différents selon les localités. Les plus fortes attaques ont été observées à Niaouli, Togoudo, Eglimé et Malanville (tableaux 1 et 2). Les sites de Koussin, Sowé et Dokparou n'ont enregistré aucune attaque en 2007 et 2008. Mais il est impossible de dire si la différence de niveau d'attaque entre les sites est liée aux caractéristiques climatiques ou à la résistance des variétés, puisque les variétés sont différentes d'un site à l'autre. La variété WAB56-50 (O. sativa), stockée pendant quatre mois dans le site de Togoudo, a été la plus attaquée avec $18 \%$ de grains attaqués. Elle a été suivie par les variétés locales $(O$. glaberrima) du site d'Eglimé avec $14 \%$ de dégât au terme de quatre mois de stockage. Les variétés les moins attaquées ont été INARIS (O. glaberrima) et Toss-Long (O. glaberrima) avec des moyennes respectives de $5 \%$ et $8 \%$, mais après seulement 2 mois de stockage. Dans l'ensemble, les dégâts enregistrés par les $O$. glaberrima ont été faibles à moyens par rapport à ceux subis par la variété $O$. sativa, WAB56-104. Parmi les $O$. glaberrima, les variétés améliorées ont subi moins de préjudices que les variétés locales. Les variétés les plus susceptibles d'être attaquées seraient celles ayant une faible teneur en amylose (Ashamo et Khanna, 2006).

La corrélation positive et hautement significative entre les populations captu-

\section{Références}

Arbogast RT, Throne JE. Insect infestation of farm-stored maize in South Carolina: Towards characterization of a habitat. J stored Prod Res $1997 ; 33$ : 187-98.

Ashamo MO, Khanna SC. Resistance to Angoumois grain moth, Sitotroga cerealella (Oliv.) in paddy varieties. Annals of Plant Protection Sciences $2006 ; 14$ : 333-7.

Barney RJ, Weston PA. Movement of Angoumois grain moth (Lepidoptera: Gelechiidae) in a small farm ecosystem. Environmental Entomology $1996 ; 25: 261-7$.

Boshra SA. Effect of high-temperature preirradiation on reproduction and mating competitiveness of male Sitotroga cerealella (Olivier) and their F1 progeny. J stored Prod Res 2007; $43: 73-8$.

CAB International. Crop Protection Compendium. Wallingford (Royaume-Uni) : CAB International, 2005. www.cabicompendium.org/cpc

Gbati O, Hell K, Azoma, Skovgaard H. Danish Pest Infestation Laboratory Annual Report (Danemark). 2002. www.dpil.dk/dpil2005/pdf/ annrep/2002/ar02c8.pdf

Hansen LS, Skovgaard H. Development time and age-specific fecundity of Sitotroga cerealella in relation to temperature and relative humidity. Annual Report. Lyngby (Danemark): Danish Pest Infestation Laboratory, 2002. www.dpil.dk/ dpil2005/pdf/annrep/2002/ar02c8.pdf

Hansen LS, Skovgard S, Hell K. Life Table Study of Sitotroga cerealella (Lepidoptera: Gelichiidae), a Strain from West Africa. Journal of Economic Entomology 2004 ; 97 : 1484-90.

MAEP/DFRN. Base des données géoréférencées sur I'utilisation agricole des terres au Bénin. Rapport final. Cotonou: CENATEL, 2000. www. fao.org/ag/agl/agll/landuse/docs/benin.doc

Nos études ont démontré la présence de $S$. cerealella sur le riz au Bénin, mais avec des niveaux de population et de dégâts très variables selon les localités, les modes de stockage, les durées de stockage et les variétés. Cependant, le dispositif d'enquête dont nous disposions, étroitement lié aux pratiques paysannes, ne nous a pas permis d'isoler l'influence des différents facteurs, même si des tendances peuvent être notées, très nettes pour l'influence de la durée de stockage.

\section{Remerciements}

Les auteurs adressent leurs sincères remerciements au Centre des ressources de biodiversité de l'IITA et à son responsable, le docteur George Gorgen, pour les travaux de vérification de l'identité de l'espèce, au docteur Kerstin Hell, pour la fourniture des pièges à phéromones, et à Mele Melchiade pour sa participation aux activités de terrain.
Nykjær M, Skovgard H. Intra- and interspecific competition between Sitophilus zeamais and Sitotroga cerealella. Lyngby (Danemark) : Danish Pest Infestation Laboratory. Annual Report (Danemark). 2002. www.dpil.dk/dpil2005/pdf/ annrep/2002/ar02c8.pdf

Raoelijaona C. Les insectes ravageurs du riz, du maïs stockés et des légumineuses sèches. Recueil des exposés lors du symposium à Toliara du 7 au 11 octobre 1996. Antananarivo: Service de la surveillance phytosanitaire du territoire national, 1996. www.fao.org/inpho/ content/documents/vlibrary/move_rep/x0298f/x0 298F13.htm

Ratnadass A, Sauphanor B. Les pertes dues aux insectes sur les stocks paysans de céréales en Côte d'Ivoire. In: Parmentier M, Foua-Bi K: Céréales en régions chaudes: conservation et transformation. Paris, Montrouge: AUPELFUREF ; John Libbey, 1989.

SAS Institute. SAS systems for Windows. Version 9.1. Cary (NC) : SAS Institute Inc, 2002-2003.

Teetes G, Reddy K, Leuschner K, House R. Manuel d'identification des insectes nuisibles au sorgho., Bulletin d'information $n^{\circ} 12$. Patancheru (Inde) : ICRISAT, 1995. 